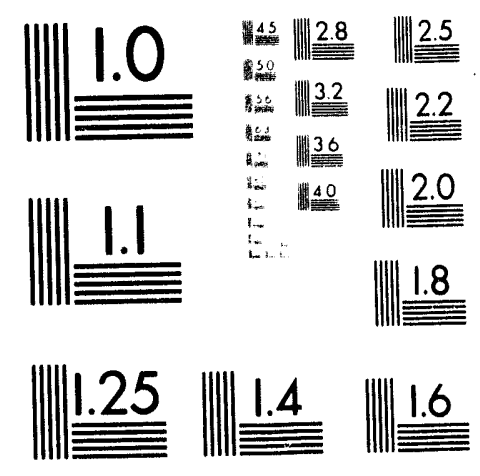



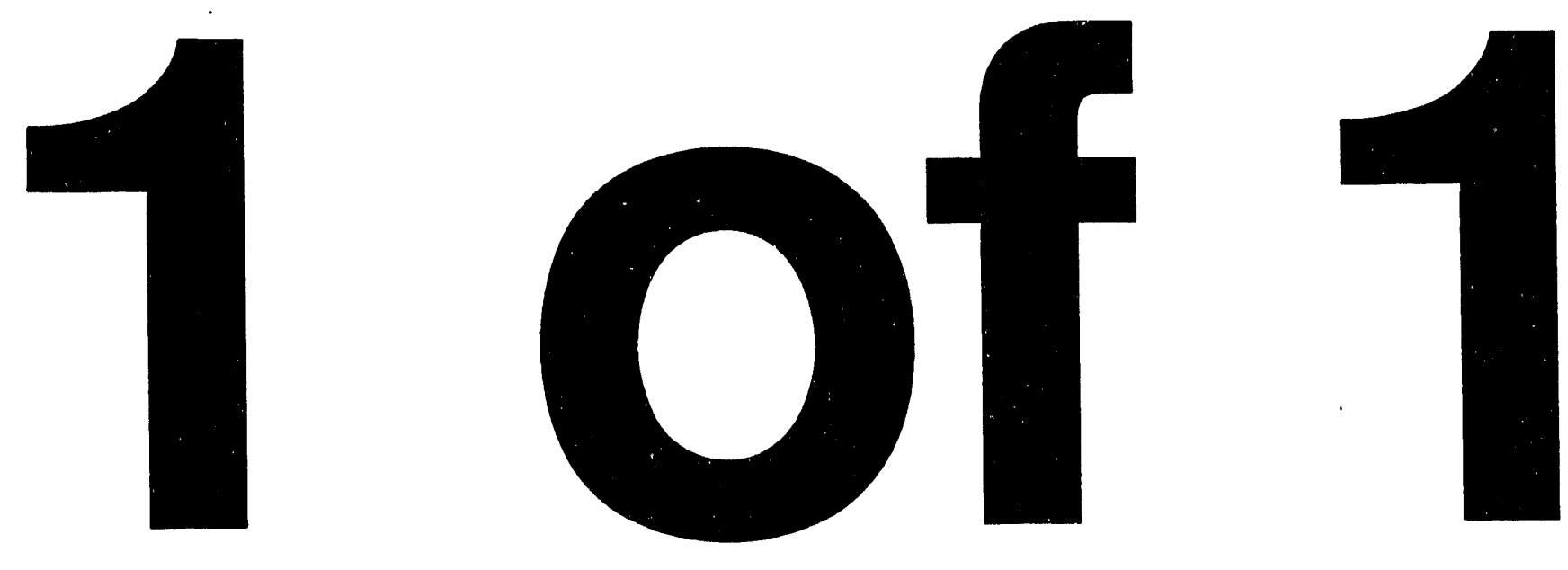


\section{INSTITUTE FOR \\ FUSION STUDIES}

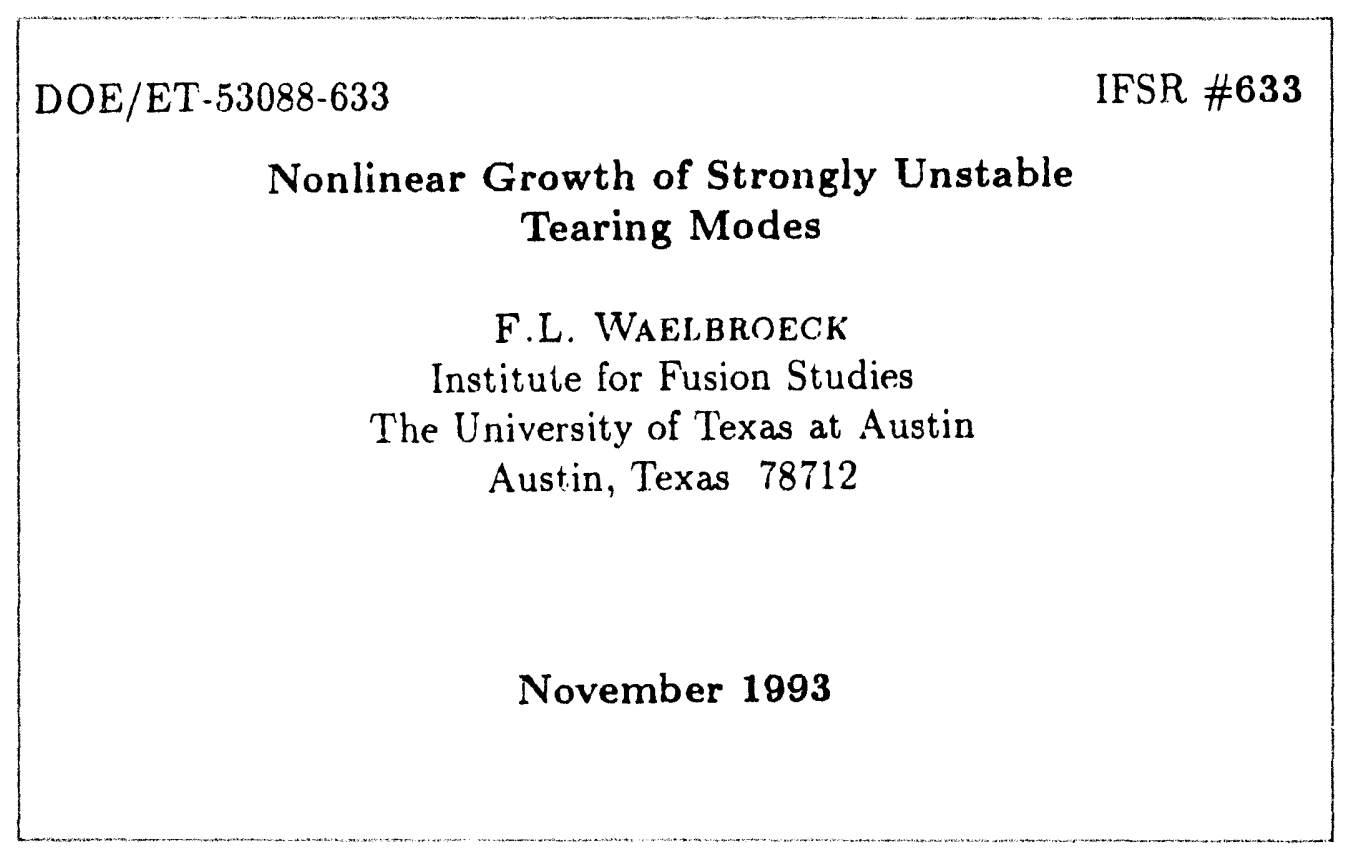

\section{THE UNIVERSITY OF TEXAS}

\section{AUSTIN}




\title{
Nonlinear Growth of Strongly Unstable Tearing Modes
}

\author{
F. L. Waelbroeck \\ Institute for Fusion Studies \\ The University of Texas at Austin \\ Austin, Texas 78712
}

\begin{abstract}
Rutherford's theory of the tearing instability is extended to cases where current nonlinearities are important, such as long wavelength modes in current slabs and the $m=1$ instability in tokamaks with moderately large aspect-ratios. Of particular interest is the possibility that the associated magnetic islands, as a result of secondary instabilities, have a singular response to the Ohmic diffusion of the current. A family of islands is used to test this possibility; it is found that the response remains bounded.
\end{abstract}

PACS numbers: $52.30 . \mathrm{Bt}, 52.30 . \mathrm{Jb}, 52.35 . \mathrm{Py}, 52.55 . \mathrm{Fa}$

\section{DISCLAIMER}

This report was prepared as an account of work sponsored by an agency of the United States Government. Neither the United States Government nor any agency thereof, nor any of their employees, makes any warranty, express or implied, or assumes any legal liability or responsibility for the accuracy, completeness, or usefulness of any information, apparatus, product, or process disclosed, or represents that its use would not infringe privately owned rights. Reference herein to any specific commercial product, process, or service by trade name, trademark, manufacturer, or otherwise does not necessarily constitute or imply its endorsement, recommendation, or favoring by the United States Government or any agency thereof. The views and opinions of authors expressed herein do not necessarily state or reflect those of the United States Government or any agency thereof. 


\section{Introduction}

The nonlinear growth of strongly unstable tearing modes is of interest in connection with theories of disruptive phenomena in laboratory (White 1986; Irby, Drake and Griem 1979; Edwards et al. 1986), space (Lui 1987; Vasilyunas 1975), and astrophysical plasmas (Coppi and Friedland 1971; Van Hoven 1979; Otani and Strauss 1988). These phenomena share two defining properties:

1. They involve rapid plasma motions.

2. They have well-defined onsets.

The first property may be accounted for by the kink-tearing mode, a hybrid of the tearing and ideal kink instabilities responsible for rapid magnetic reconnection (Otani and Strauss 1988; Kadomtsev 1975; Park, Monticello and White 1984; Aydemir, Ross and Wiley 1989; Sato et al. 1989; Waelbroeck 1989). In order to account for the second property, however, it is necessary to understand the processes taking place before the onset of the kink-tearing mode, when reconnection is ruled by the slniver, diffusion-dominated tearing mode (Rutherford 1973; Steinolfson and Van Hoven 1984). This is the subject of the present paper.

In a recent letter (Waelbroeck 1993, henceforth I), a singularity in the nonlinear evolution of robust tearing instabilities was described and proposed as a trigger for the onset of the kink-tearing mode. This letter was based on exact solutions for the spatial structure of the mode, but used a phenomenological model to describe the temporal evolution of the island. The object of the present paper is to derive an exact evolution equation for strong tearing modes and to describe the properties of these modes.

Strong tearing modes are defined here by

$$
\lambda \Delta^{\prime} \gg 1
$$


where $\lambda$ is the wavelength of the mode and $\Delta^{\prime}$ is the stability parameter defined by Furth, Killeen and Rosenbluth (1963). Furth, Rutherford and Selberg have shown that this $\Delta^{\prime}$ parameter, which has the dimensions of inverse length, can be interpreted as a measure of the free energy available to the instability. It relates the total current perturbation to the reconnected flux $\tilde{\psi}$ by

$$
\int \tilde{J} d x=\Delta^{\prime} \tilde{\psi}
$$

where $\tilde{J}$ is the current-density perturbation and $x$ is the transverse coordinate. The strong tearing criterion results in the appearence of a new regime at small island amplitude,

$$
w \sim 1 / \Delta^{\prime} \ll \lambda
$$

This new, strong tearing regime differs from the ordinary tearing regime $\left(w \Delta^{\prime} \ll 1\right)$ in several respects:

1. Diffusive Evolution: For ordinary tearing modes, the current diffuses more rapidly than the flux and thus remains in diffusive equilibrium during reconnection. In the strong tearing regime, by contrast, the current and flux diffusion rates are comparable and the current evolves dynamically during reconnection. This may be seen by substituting the current obtained from Ohm's law, $\tilde{J} \sim(\partial \tilde{\psi} / \partial t) / \eta$, into Eq. (1). There follows the reconnection time $\tau_{\psi}=\tilde{\psi} /(\partial \tilde{\psi} / \partial t) \sim w /\left(\eta \Delta^{\prime}\right)$. Comparing this to the characteristic time for current diffusion, the island skin time $\tau_{J}=w^{2} / \eta$, one finds

$$
\tau_{J} / \tau_{\psi} \sim w \Delta^{\prime}
$$

Note that the nonlinear regime describes islands such that the characteristic diffusion time is small compared to the period of shear-Alfvén oscillations: this justifies an equilibrium analysis of the magnetic configuration.

2. Equilibrium: In the strong tearing regime, the current-density perturbation is comparable to the equilibrium current-density. Their ratio can be evaluated from Eq. (1) 
and the estimate $\tilde{\psi} \sim J_{0} w^{2}$, where $w$ is the island width. One finds

$$
\tilde{J} / J_{0} \sim w \Delta^{\prime}
$$

This implies that the constant-psi approximation underlying Rutherford's classical analysis of nonlinear tearing modes (1973) fails in the strong tearing regime: the more general theory described in I must then be used.

3. Stability: The magnitude of the current filamentation in the strong tearing regime affects the stability of the island against current driven, kink-like secondary instabilities. These may constitute a triggering mechanism distinct from that described in I. The stability question is addressed here by searching for singularities in the response of the island to the Ohmic evolution in the current profile. A more general method for evaluating the stability of magnetic islands will be described in a future publication.

The preceding considerations clearly distinguish the strong tearing regime from previously known regimes. One is led to a classification of nonlinear reconnection into the ordinary tearing, strong tearing, and resistive-kink regimes. In linear theory, by comparison, only two regimes are distinguished. The relevant parameter is $w_{i} \Delta^{\prime}$, where the inertial layer width $w_{i}=\eta^{1 / 3} k_{\|}^{1 / 3}$ is defined so that the local Alfvén time $\tau_{A}=k_{\|}^{\prime} w_{i}$ equals the layer's skin time $\tau_{R i}=w_{i}^{2} / \eta$ (here $k_{\|}^{\prime}$ is the radial derivative of the parallel component of the wavevector). For $w_{i} \Delta^{\prime} \gg 1$ the instability has rapid growth: $\gamma \sim k_{\|}^{2 / 3} \eta^{1 / 3}$. This is the linear kink-tearing mode described by Coppi et al. (1976). For $w_{i} \Delta^{\prime} \ll 1$, by contrast, the ordinary tearing mode of Furth, Killeen and Rosenbluth (1963) is obtained with $\gamma \sim k_{\|}^{2 / 5} \eta^{3 / 5} \Delta^{14 / 5}$ (Steinolfson and Van Hoven 1983). The distinction drawn above between strongly unstable and ordinary tearing modes has thus no analog in linear theory. Note that islands initially in the linear kink-tearing regime will remain in the kink-tearing regime nonlinearly as shown by Baty, Luciani and Bussac (1991). The present analysis addresses the cases where either 
$w_{i} \ll 1 / \Delta^{\prime} \ll \lambda$ (e.g. in moderate aspect ratio, high temperature tokamaks), or where a nonlinear island $\left(w>w_{i}\right)$ is present in the initial state.

The remainder of this paper is organized as follows. First, the representation of thin island equilibria is reviewed in Sec. II. The quasi-equilibrium form of Ohm's law is next derived in Sec. III. It is used in Sec. IV to obtain the evolution equations for thin islands. The response of magnetic islands to changes in the current-density profile is investigated in Sec. V, and the conclusions are stated in Sec. VI.

\section{Thin Island Equilibrium}

\section{A. Formulation}

The analysis is based on the low-beta reduced magnetohydrodynamic (MHD) equations. The magnetic field is taken to be helically symmetric as well as periodic in the longitudinal direction. It may conveniently be separated into a reference field consisting entirely of closed field-lines everywhere parallel to the symmetry direction, and a residual field $\mathbf{B}_{*}$ called the auxiliary field:

$$
\mathbf{B}=\widehat{\mathbf{z}}+\epsilon\left(r \hat{\boldsymbol{\theta}}-\mathbf{B}_{*}\right)
$$

where $\epsilon$ is the inverse aspect-ratio and $\widehat{\mathbf{z}}, \hat{\boldsymbol{\theta}}$ are respectively the toroidal and poloidal unit vectors. The auxilliary field may be expressed in terms of the flux $\psi$ through a helical ribbon as $\mathbf{B}_{*}=\hat{\mathbf{z}} \times \nabla \psi$.

The current-density $J$ is related to the helical flux by Ampére's law, $J=\nabla^{2} \psi-2$. In equilibrium, $\mathbf{B} \cdot \boldsymbol{\nabla} J=0$ so that $J$ is constant on flux-surfaces. Let $J=I(\psi)-2$. The local equilibrium equation then takes the form of a generalized Liouville equation,

$$
\nabla^{2} \psi=I(\psi)
$$

the ordinary Liouville equation being recovered for exponential current distributions $I$. For thin islands, the Laplacian may be approximated by $\partial^{2} \psi / \partial x^{2}$, where $x=r-r$, and $r$, 
is the radius of the $q=1$ resonant surface. A closed-form solution may then obtained by quadrature (Bernstein, Greene and Kruskal 1957; Waelbroeck 1989, 1993).

\section{B. Magnetic Field}

The first integration yields an expression corresponding to the Biot-Savart law for the longitudinal component of the auxiliary field,

$$
B_{* \theta}= \pm[2(F(\psi)-G(\theta))]^{1 / 2}
$$

where $F(\psi)$ represents the total current contained within the flux surface(s) $\psi$ :

$$
F(\psi)=F\left(\psi_{o}\right)+\int_{\psi_{0}}^{\psi} I(\hat{\psi}) d \hat{\psi}
$$

Here $\psi_{o}$ is the value of the helical flux on the island's magnetic axis. The two solution branches in Eq. (3) must be connected at their turning-point where $B_{* \theta}=0$, or $F\left(\psi_{t}\right)=G(\theta)$ (Fig. 1). $G(\theta)$ may thus be interpreted as the current contained within the flux surface $\psi_{t} \tan$ gent to the radial chord at $\theta$. Flux surfaces are divided into reconnected or "trapped" surfaces with $F\left(\psi_{s}\right)<\operatorname{Max}[G(\theta)]$ and unreconnected, "circulating" surfaces with $F\left(\psi_{0}\right)>\operatorname{Max}[G(\theta)]$. The separatrix is the last flux-surface to have a turning-point: $F\left(\psi_{0}\right)=\operatorname{Max}[G(\theta)]$. The $x$-point will henceforth be assumed to lie at $\theta=0$, and the constant of integration in $F$ and $G$ will be fixed by $G(0)=\operatorname{Max}[G(\theta)]=0$. Reconnected flux surfaces will thus be labeled by $F<0$ and unreconnected surfaces by $F>0$.

\section{Flux Surface Displacement}

The transverse displacement of the flux-surfaces is next determined by a second integration:

$$
x_{ \pm}(\psi, \theta)=x_{t}(\theta) \pm \int_{\psi_{t}}^{\psi} \frac{d \psi}{B_{* \theta}},
$$

where $x_{t}(\theta)$ represents the position of the turning point. Note that current-density asymmetries may be represented by allowing the function $F(\psi)$ to have two branches outside of the island. In what follows, however, we will only consider the case $F_{+}(\psi)=F_{-}(\psi)$. 
The equilibrium problem is closed by matching each harmonic of the solution in the island region, Eq. (4), to the corresponding solution of the linearized toroidal MHD equation away from the island. There follows the global equilibrium equations

$$
\int_{\psi_{0}}^{\infty} d \psi c_{l}(\psi)=-\frac{\pi}{2} \frac{\Delta_{l}^{\prime} G_{c l}}{I_{0}^{2}}
$$

for $l=1,2, \ldots$, where $G_{c l}$ is the $\mathrm{n}$-th cosinusoidal Fourier coefficient of $G(\theta)$ and

$$
c_{l}=\langle\cos (l \theta)\rangle .
$$

The angular brackets are defined by

$$
\langle h(\psi, \theta)\rangle=\int_{\theta_{\mathrm{t}}}^{2 \pi-\theta_{\mathrm{t}}} d \theta \frac{h(\psi, \theta)}{B_{* \theta}},
$$

where for reconnected flux surfaces $(F<0), \theta_{t}$ is the turning point determined implicitly by $G\left(\theta_{t}\right)=F(\psi) ;$ for unreconnected surfaces $(F>0), \theta_{t}=0$. Note that the bracket operation is identical to the flux-surface average for $F>0$ but is only half of the flux-surface average for $F<0$. In the case of asymmetric islands the system must be completed by a set of homologous equations for the sinusoidal Fourier coefficients $G_{\imath l}$. Henceforth even parity will be assumed unless otherwise indicated, and the notation $G_{c l}=G_{l}$ will be adopted.

For some applications it is more convenient to write the equilibrium equation in terms of the azimuthal variation of the core displacement $\xi_{\infty}$ given by the limit of $x_{ \pm}(\psi, \theta)-x_{0}(\psi)$ away from the island. Here $x_{0}(\psi)$ is the position of the flux surface $\psi$ in the unreconnected reference state. From the inner solution, Eq. (4), the core displacement is found to be

$$
\xi_{\infty}(G)=2 \int_{G_{\min }}^{\infty} d F \frac{\partial \psi}{\partial F}\left(\frac{H(F-G)}{[2(F-G)]^{1 / 2}}-\frac{1}{2 \pi} \oint d \theta \frac{H(F-G)}{[2(F-G)]^{1 / 2}}\right) .
$$

This representation has the advantage that $\xi_{\infty}$ depends on the azimuthal angle solely through $G(\theta)$. The matching conditions, or global equilibrium equations (5), determine $\xi_{\infty}(G)$ in terms of the Fourier components of $G(\theta)$ :

$$
\xi_{\infty}(G)=-I_{0}^{-2} \sum_{l=1}^{\infty} \Delta_{l}^{\prime} G_{l} \cos l \theta
$$


It was shown in I that the global equilibrium equations (5), or equivalently Eqs. (8)-(9), completely determine the magnetic configuration for a given current-well $G(\theta)$ and external flux distribution $\psi(F), F>0$. Explicit solutions of these equations were constructed for a family of model current distributions. Note that there is no $l=0$ component of the equilibrium equation: this reflects the freedom to choose the current profile.

\section{Constant-Psi Limit}

For weak current perturbations, $\tilde{I}(\psi)=I(\psi)-I_{0} \ll I_{0}$, an expansion in powers of $\tilde{I} / I_{0}$ can be used to simplifv the analysis. To lowest order, the current distribution is $F(\psi) \simeq I_{0}\left(\psi-\psi_{s}\right)$ and Eq. (3) becomes

$$
B_{* \theta}= \pm\left[2 I_{0}\left(\psi-\psi_{s}-G(\theta) / I_{0}\right)\right]^{1 / 2},
$$

Alternatively, direct integration of the equilibrium equation $\partial^{2} \psi / \partial x^{2}=I_{0}$ yields $B_{* \theta}=$ $\partial \psi / \partial x=I_{0} x$. These two expressions for $B_{* \theta}$ may be used to solve for $\psi$ :

$$
\psi(x, \theta)=I_{0} x^{2} / 2+\psi_{s}+G(\theta) / I_{0}+O\left(\left(\tilde{I} / I_{0}\right) \psi_{\mathbf{s}}\right)
$$

The first term in this equation is evidently the unperturbed flux distribution, $\psi_{0}(x)=I_{0} x^{2} / 2$. The subsequent terms constitute the perturbation, $\tilde{\psi}=\psi_{\mathbf{s}}+G(\theta) / I_{0}$. Note that $\tilde{\psi}$ does not vary in the transverse direction: $\partial \tilde{\psi} / \partial x=0$. For this reason the weak current-perturbation expansion is unfortunately known as the constant-psi approximation, although this name fails to convey its physical nature.

The equilibrium equations are easily simplified with the change of integration variable $\psi \rightarrow F$. The corresponding Jacobian is the inverse current-density $\partial \psi / \partial F \simeq I_{0}^{-1}\left(1-\tilde{I} / I_{0}\right)$. Integrating term by term, the iowest order integrals are found to vanish. The first order equations are then

$$
\int_{G_{\min }}^{\infty} d F \tilde{I}(\psi) c_{l}(\psi)=\frac{\pi}{2} \Delta_{l}^{\prime} G_{c l},
$$

This is simply the formal expression of Eq. (1). 
In the following section, the general equilibrium equation, $J=I(\psi)-2$ will be used to derive the quasi-equilibrium form of Ohm's law. The results of the present section will be used in Sec. IV, in conjunction with the quasi-equilibrium form of Ohm's law, to obtain the island evolution equations.

\section{Equilibrium Ohm's Law}

In the presence of dissipation, magnetofluids generally evolve through continuous sequences of states close to mechanical equilibrium: force-balance remains approximately satisfied throughout the evolution, and inertia has a negligible effect. When this is the case, it is desireable to eliminate the fluid velocity from the system of equations describing the evolution, so as to obtain a closed system of equations for the flux-surface quantities determining the equilibrium (Grad and Hogan 1970; Taylor 1975). For tearing modes, the relevant transport equation is Ohm's law,

$$
\frac{\partial \psi}{\partial t}+\mathbf{v} \cdot \boldsymbol{\nabla} \psi=\eta J-E_{0}
$$

The perpendicular fluid velocity is related to the electric field by the usual MHD condition $\mathbf{v}=\widehat{\mathbf{z}} \times \boldsymbol{\nabla} \phi$. Equation (10) may thus be expressed as a magnetic differential equation for the electrostatic potential,

$$
\text { B. } \boldsymbol{\nabla} \phi=\eta J-E_{0}-\frac{\partial \psi}{\partial t} \text {. }
$$

The solubility condition for this equation is

$$
\langle\partial \psi / \partial t\rangle=\left(\eta I(\psi)-E_{* 0}\right)\langle 1\rangle
$$

where $E_{* 0}=E_{0}-2 \eta$. The equilibrium property was used here to factor the current from the flux-surface average. The solubility condition distills all the information provided by Ohm's law regarding the evolution between neighboring equilibria. In orcier for Eq. (11) to be of practical utility, however, the time derivative must be commuted with the flux-surface average operation. There are several ways of accomplishing this: 
In Rutherford's theory, the average of the inductive electric field perturbation, $\langle\partial \psi / \partial t\rangle$, is evaluated by extending the constant-psi approximation to the time-derivative of the flux perturbation. That is,

$$
\frac{\partial \psi}{\partial t}=\sum_{l=1}^{\infty} \frac{d \tilde{\psi}_{l}}{d t} \cos l \theta
$$

where the $d \psi_{l} / d t$ coefficients are assumed to be spatially constant. This allows the current perturbation to be evaluated in terms of the rates of reconnection:

$$
\tilde{I}(\psi)=\frac{1}{\eta} \sum_{l=1}^{\infty} \frac{c_{l}}{c_{0}} \frac{d \tilde{\psi}_{l}}{d t} .
$$

Note that this is the inverse of the normal solution procedure: Instead of calculating the reconnection rate for a given initial current profile $\tilde{I}(\psi)$, one calculates the current profile in terms of the reconnection rate! Since the reconnection rate is itself deterinined by the initial geometry (in the form of the $\tilde{\psi}_{l}$ ), the ability to specify the initial current profile has been lost. The reason for this will become clear in Sec. IV.

In the strong tearing regime, $\langle\partial \psi / \partial t\rangle$ can be calculated by direct application of the thin island formalism. This requires writing the inductive electric field in terms of the flux surface displacement, $\partial \psi / \partial t=B_{* \theta}(\partial x / \partial t)_{\psi, \theta}$, and using the results of the previous section to calculate the corresponding flux-surface averages. A more general and elegant approach, however, is to use Ohm's law to derive an evolution equation for the magnetic helicity distribution.

It is convenient, within the context of large aspect-ratio, helically symmetric geometry, to redefine the local magnetic helicity by $\mathcal{H}(\psi)=d \Phi / d \psi$, where $\Phi(\hat{\psi})$ is the toroidal flux within the surface(s) of helical flux $\hat{\psi}$ :

$$
\Phi(\widehat{\psi})=\int_{-\infty}^{+\infty} d x \oint d \theta H(\psi(x, \theta)-\widehat{\psi}) .
$$

Here $H$ is the usual Heaviside function. The helicity is then given by

$$
\mathcal{H}(\hat{\psi})=-\int_{-\infty}^{+\infty} d x \oint d € \delta(\psi(x, \theta)-\hat{\psi}) .
$$


As remarked by Kadomtsev (1977) however, this definition differs from the usual definition of magnetic helicity (Taylor 1974),

$$
\mathcal{K}=\int d V \mathbf{A} \cdot \mathbf{B}
$$

where the integral extends over a tube of flux. The two definitions are related by $\mathcal{H}=$ $\psi \mathcal{K}+O(\epsilon)$. Note that $\mathcal{H}$ is analogous to the safety factor: $q=d \Phi / d \chi$, where $\chi$ is the poloidal flux. Clearly, both quantities measure the winding density of field lines around their magnetic axes.

The local helicity, most importantly, provides a natural formulation of the equilibrium limit of Ohm's law. Consider the time derivative of Eq. (13),

$$
\frac{\partial \mathcal{H}}{\partial t}=\int_{-\infty}^{+\infty} d x \oint d \theta \frac{\partial \psi}{\partial t} \delta^{\prime}(\psi(x, \theta)-\widehat{\psi})
$$

The right-hand side of this expression may be evaluated by using Ohm's law, Eq. (10). Changing the integration variables to $\psi, \theta$ and using the jacobian $\mathcal{J}=B_{* \theta}^{-1}$ there follows, after integration by parts,

$$
\frac{\partial \mathcal{H}}{\partial t}=-\frac{\partial}{\partial \psi}\left[\left(\eta I-E_{* 0}\right) \mathcal{H} j\right.
$$

Equation (14) is the basic Ohmic evolution equation for helically symmetric equilibria. Note that this result is independent of the thin-island approximation.

The application of Eq. (14) to thin islands is the subject of the following section.

\section{Thin Island Evolution}

The thin-island evolution equations are now obtained from Eq. (14) by straightforward application of the results of Sec II. The magnetic helicity is found to be $\mathcal{H}=-c_{0}(F ; G(\cdot))$, where the functional dependence of $c_{0}$ on $G(\cdot)$ is indicated by the semicolon. It is convenient to use $F$ as the independent transverse variable, so as to keep the $c_{l}$ independent of the 
current distribution. The evolution equations are then

$$
\left.\frac{\partial \psi}{\partial t}\right|_{F}+\left.\frac{\partial \psi}{\partial F}\right|_{t} \sum_{l=1}^{\infty} \frac{c_{l}^{\prime}-c_{0}^{\prime}}{c_{0}^{\prime}} \frac{d G_{l}}{d t}=\Omega(F, t ; \psi),
$$

where $c_{l}^{\prime}=d c_{l} / d F$ (for fixed $\left.G(\cdot)\right)$ and the time derivative is to be taken at constant $F$. The first two terms in this equation represent the convective derivative of the flux distribution. The right-hand side term, $\Omega$, represents the Ohmic diffusion of the flux:

$$
\Omega(F, t ; \psi)=\frac{1}{c_{0}^{\prime}} \frac{\partial}{\partial F}\left[c_{0}\left(\eta\left(\frac{\partial \psi}{\partial F}\right)^{-1}-E_{* 0}\right)\right] .
$$

The evolution of the current-well $G(\cdot)$ is determined by taking the time derivative of the equilibrium equation and using Eq. (15) to eliminate the rate of evolution of the flux distribution. There follows

$$
\sum_{l=1}^{\infty} a_{k, l} \frac{d G_{l}}{d t}=b_{k}
$$

where

$$
\begin{aligned}
a_{k, l} & =\frac{\pi}{2} \frac{\Delta_{k}^{\prime}}{I_{0}^{2}} \delta_{k, l}+\int_{G_{\min }}^{\infty} d F \frac{\partial \psi}{\partial F} \alpha_{k, l} ; \\
\alpha_{k, l} & =c_{k}^{\prime} c_{l}^{\prime} / c_{0}^{\prime}-\left(c_{k+l}^{\prime}+c_{k-l}^{\prime}\right) / 2 ; \\
b_{k} & =-\left(\eta\left(\frac{\partial \psi}{\partial F}\right)^{-1}-E_{* 0}\right)\left(1-(-1)^{k}\right) c_{0}\left(G_{\min }\right)+\int_{G_{\min }}^{\infty} d F \beta_{k} ; \\
\beta_{k} & =\left(c_{k}^{\prime}-c_{0}^{\prime}\right) \Omega(\psi ; F, t) .
\end{aligned}
$$

Two boundary conditions must now be specified, since $\Omega$ depends on the second derivative of the flux distribution. A first condition follows by requiring that the current-density match the unperturbed current-density away from the island,

$$
\lim _{F \rightarrow \infty} \frac{\partial \psi}{\partial F}=I_{0}^{-1}
$$

A second boundary condition is obtained by observing that the helicity describes flux tubes, and cannot carry information concerning the magnetic axis, the latter being a bounding 
field-line. It follows that the expression of Ohm's law on the magnetic axis is independent of Eq. (15) and may serve as the second boundary condition:

$$
\frac{\partial \psi}{\partial t}+\frac{\partial \psi}{\partial F} \frac{d G_{\min }}{d t}=\eta\left(\frac{\partial \psi}{\partial F}\right)^{-1}-E_{* 0}
$$

Equations (15)-(16), with the boundary coiditions (17)-(18), completely specify the evolution of strongly unstable tearing modes from a given initial state. These equations, unlike those of Rutherford (1973), do not enjoy a scale invariance property. The lack of scale invariance is clearly necessary for a theory of disruptive processes, but precludes finding analytic solutions. Useful information may nevertheless be obtained from the structure of the evolution equations. In the following two subsections, the nature of the singularities and the constant-psi limit of these equations are examined. The regularity of the inverse of the matrix $a$, which may be interpreted as the response of the island to the evolution of the flux distribution $\psi(F)$, will be investigated in the next section.

\section{A. Interpretation of the Singularities}

Three types of singularities can be distinguished in Eqs. (15)-(16), each corresponding to a different physical circumstance. All these singularities were found to be regular for wellbehaved $\psi(F)$ and $G(\theta)$ distributions (Appendix I).

The first type of singularity appears in the coefficients $c_{l}$ and corresponds to the vanishing of the longitudinal magnetic field at the turning points. It is integrable, and is unrelated to any of the approximations in the theory.

The second type of singularity, found in the integrands $\alpha_{k, l}$ and $\beta_{k}$, results from the vanishing of the local shear-Alfvén velocity at the separatrix and the consequent failure of the equilibrium approximation. The separatrix singularities are physically resolved by inertia. Since they are integrable, however, the global evolution will be little affected by inertial layer dynamics. This conclusion is analogous to that for constant-psi islands, where the inertial corrections have been shown to be small by Edery et al. (1983). 
The last singularity resides in the short-wavelength behavior of the $\Delta^{\prime}$ coefficients, $\Delta_{l}^{\prime} \sim$ $2 l / r_{s}$. It is associated with the breakdown of the thin-island approximation $(\partial / \partial x \gg \partial / \partial \theta)$ at these wavelengths. For sufficiently smooth islands, $G_{l}=o(1 / l)$, the affected terms are well behaved.

\section{B. Constant-Psi Limit}

To establish the connection with Rutherford's theory, consider the limit ui weak current perturbations. Unexpectedly, Eqs. (15)-(18) remain more general than those of Rutherford even in this limit. Most noticeably, the current (given implicitly by $\psi(F)$ ) is now determined dynamically by Eq. (15), instead of statically as in Eq. (12). Although the reconnection rates are similar, Eq. (15) shows that the current distribution evolves at a rate proportional to the inverse of the island skin-time $\tau_{s}=w^{2} / \eta$ (Appendix II).

Since the rate of current evolution exceeds the reconnection rate by a factor of $1 / w \Delta^{\prime}, \tilde{\psi}$ is approximately constant during the first few island skin-times. There is thus no significant contribution of external free energy during the initial evolution and the current distribution will relax dissipatively into a diffusive quasi-equilibrium. It is this relaxed diffusive quasiequilibrium which is described by Rutherford's theory.

During the relaxation, transverse variations may be neglected in $\tilde{\psi}_{l}$ but not in $\partial \tilde{\psi}_{l} / \partial t$; whereas the current perturbation is small, its time derivative is significant. After the relaxation, by contrast, $\partial \tilde{\psi}_{l} / \partial t$, as well as $\tilde{\psi}_{l}$, is approximately constant across the island. The current, and hence the flux distribution, may then be determined from Eq. (12). It is easy to verify that this current profile is such that, to lowest order, the Ohmic term balances the convective term in Eq. (15). For comparison, the reconnection rates for Rutherford's regime are given by Eq. (16) with the matrix coefficients

$$
a_{k, l}^{R}=\int_{G_{\min }}^{\infty} d F \alpha_{k, l}^{R}
$$




$$
\alpha_{k, l}^{R}=c_{k} c_{l} / c_{0}
$$

and

$$
b_{k}^{R}=-\eta \frac{\pi}{2} \frac{\Delta_{k}^{\prime} G_{k}}{I_{0}^{2}} .
$$

In Appendix II, the limit of weak current-perturbations is considered in greater detail and Rutherford's equation is derived directly from Eqs. (15)-(18).

\section{Island Response}

The most interesting feature of the evolution equation (16) is that the rates $d G_{l} / d t$ depend on the inverse of the matrix $a$. This inverse represents the response of the island to the helicity sources (or sinks) constituted by Ohmic diffusion. If $a$ has a nuli eigenvector,

$$
\sum_{l=1}^{\infty} a_{k, l} V_{l}=0,
$$

the response is resonant, or singular. The resonance condition may be written symbolically

$$
\operatorname{det}(a)=0
$$

The relationship between resonance and instability of the magnetic islands is somewhat complicated. It can be summarized by saying that first, resonance implies the existence of nearby unstable equilibria but second, it is not necessary for instability.

The first statement follows from the principle of exchange of stability and the observation that the null current-well perturbation $\delta G_{l}=V_{l}$ and the associated ideal perturbation of the flux distribution,

$$
\delta \psi=-\frac{\partial \psi}{\partial F} \sum_{l=1}^{\infty} \frac{c_{l}^{\prime}-c_{0}^{\prime}}{c_{0}^{\prime}} V_{l},
$$

constitute a neutrally stable perturbation of the equilibrium: By construction, they satisfy the linearized equilibrium equation and the ideal MHD constraints.

The second assertion results from the observation that the representation of the perturbation by $\{\delta \psi(F), \delta G(\theta)\}$ is too restrictive to allow the detection of a general stability 
transition. Recall that in cylindrical equilibria, neutrally stable perturbations have discontinuous longitudinal magnetic fields at the mode-rational surface. In magnetic islands, by analogy, a discontinuity is to be expected at the separatrix and possibly at the $\mathrm{O}$-point. The perturbation $\{\delta \psi(F), \delta G(\theta)\}$, however, can only describe constant-amplitude current sheets (as a plateau in $\delta \psi(F)$ ), corresponding to azimuthally constant jumps in the square of the longitudinal field.

More generally, it is important to bear in mind that the global equilibrium equation is in fact a condition for smooth matching between the local solutions of the equilibrium equation in the interior and exterior regions. As a result, a choice of two functions $\psi(F)$ and $G(\theta)$ corresponds to a well-behaved island only if these functions satisfy the global equilibrium equation. This is an inherent limitation of the thin-island representation as concerns stability analysis: It can only describe neutrally stable perturbations. In particular, it is not possible to construci a $\delta W$ variational principle solely in terms of $\delta \psi(F), \delta G(\theta)$.

Despite these limitations, the resonance condition remains a useful stability diagnostic. It is also of interest for its possible role in the tearing singularity described in I. Two applications will be made below.

\section{A. Odd Modes: Self-Coalescence Instability}

The resonance condition may be investigated analytically in the case of perturbations with odd parity in $\theta$, such as the self-coalescence instability (Bussac et al. 1984; Lee et al. 1985; Jensen and McClain 1986). For odd perturbations the ideal limit of Ohm's law $(\Omega=0)$ yields $\delta \psi(F)=0$, by virtue of $s_{l}=\langle\sin (l \theta)\rangle=0$. Instead of solving the nullvector equation (19), it is easier and equivalent to consider the linearization of the global equilibrium equation in its azimuthal representation, Eqs. (8)-(9). The significant feature of Eq. (9) is that $\xi_{\infty}$ depends on $\theta$ only through $G$. The linearized, perturbed equilibrium equation is then

$$
\frac{d \xi_{\infty}}{d G} \delta G=-\frac{1}{2} \Delta_{1}^{\prime} \delta G_{s 1} \sin \theta
$$


substituting the value of $\xi_{\infty}$ from the reference equilibrium equation (9), and neglecting the higher order $\Delta_{l}^{\prime}$ terms, $l>1$ yields

$$
\delta G(\theta)=-\frac{\delta G_{s 1}}{G_{1}} \frac{d G}{d \theta} .
$$

The only resonant odd perturbation of a symmetric island is thus the trivial rigid shift displacement. In particular, the self-coalescence mode does not lead to finite island-width resonances. This is consistent with the results of Bussac et al. (1984).

\section{B. Even Modes: Numerical Result;}

For even perturbations, the null-vector equations must be solved numerically. The response is generally bounded $(|\operatorname{det}(a)|>0)$, so that the search for a resonant island must encompass continuous families of islands. Fortunately, the equilibrium equation is invariant under the transformation $G \rightarrow \mu G$ and $I_{0} / I(F)-1 \rightarrow \mu^{-1 / 2}\left(I_{0} / I(F / \mu)-1\right.$ ) (recall that $I(F)=$ $\left.(\partial \psi / \partial F)^{-1}\right)$. This property makes it possible to solve the resonance condition directly for the critical island amplitude, if one exists.

It is convenient to define the normalized current distributions $g(\theta)=G(\theta) /\left|G_{\min }\right|$ and $\hat{\imath}(f)=\left(I_{0} / \Delta_{1}^{\prime}\left(-G_{\min }\right)^{1 / 2}\right)\left(I_{0} / I\left(\left|G_{\min }\right| f\right)-1\right)$, where $\{G(\theta), I(F)\}$ is a particular solution of the equilibrium equations. These normalized distributions satisfy

$$
2 \int_{-1}^{\infty} d f \hat{\imath}(f)\left(\frac{H(f-g)}{[2(f-g)]^{1 / 2}}-\frac{1}{2 \pi} \oint d \theta \frac{H(f-g)}{[2(f-g)]^{1 / 2}}\right)=-g_{1} \cos \theta
$$

where the harmonic $\Delta_{l}^{\prime}$ coefficients were neglected in favor of the fundamental. A family of islands parametrized by the depth of the current-well $\left|G_{\min }\right|$ is then given by $G(\theta)=$ $\left|G_{\min }\right| g(\theta)$ and $I_{0} / I(F)-1=\Delta_{1}^{\prime}\left|G_{\min }\right|^{1 / 2} I_{0}^{-1} \hat{\imath}\left(\left|G_{\min }\right| f\right)$. The elements of the $a$ matrix for these islands are

$$
a_{k, l}=\Delta_{1}^{\prime} I_{0}^{-2} \widehat{a}_{k, l}+\left|G_{\min }\right|^{-1 / 2} I_{0}^{-1} \bar{a}_{k, l},
$$

where

$$
\hat{a}_{k, l}=\frac{\pi}{2} \delta_{k, l}+\int_{-1}^{\infty} d f \hat{\imath}(f) \alpha_{k, l}
$$




$$
\bar{a}_{k, l}=\int_{-1}^{\infty} d f \alpha_{k, l}
$$

In terms of these matrices the null-vector equations are

$$
\sum_{l=1}^{\infty}\left(\hat{a}_{k, l}-\mu \bar{a}_{k, l}\right) V_{l}=0
$$

where $\mu=\Delta_{1}^{\prime}\left|G_{\min }\right|^{1 / 2} / I_{0}$. The resonant amplitude, if one exists, is then determined by simultaneous diagonalization of $\hat{a}$ and $\bar{a}$.

A truncated version of Eq. (20) was solved numerically for the equilibria described in I. These are specified by the choice $g(\theta)=(\cos \theta-1) / 2$ and $\imath(f)=\hat{A}\left(1+f / f_{w}\right)^{-3 / 2}, f>0$. The amplitude of the current perturbation, $\hat{A}$, is then determined by the condition of global helicity conservation obtained by integrating Ohm's law over the entire island region. One finds $\hat{A}=-\left(8 f_{w}\right)^{-1 / 2}$.

The largest eigenvalue was found to converge rapidly with the order of the truncation $N$. For $N=8$, this eigenvalue varied from -1.25 for $f_{w}=.5$ to -1.20 for $f_{w}=1.5$. Thus, for positive $\Delta_{1}^{\prime}$, no neutrally stable islands are found. It is concluded that the family of equilibria found in I does not contain a resonant equilibrium.

\section{Conclusions}

Two mechanisms may be invoked, within the context of single-helicity theory, to explain the onset of the resistive-kink mode. The first depends on the occurrence of a secondary instability as a magnetic island reaches a critical width (Finn and Kaw 1977; Bussac and Pelat 1987). The rapid evolution of the primary tearing mode accounts here for the abruptness of the onset. The second mechanism consists of a singularity in the diffusive evolution of the primary tearing mode itself (Waelbroeck 1993). This singularity, associated with a divergence of the current-density on the separatrix, follows from the conservation of the total helicity within the island region. 
In order to discriminate between these two mechanisms, a new set of evolution equations describing the nonlinear growth of robust tearing modes has been derived. These new evolution equations depend on a response operator which may exhibit resonances corresponding to neutrally stable perturbations of the equilibrium. The response remains bounded, however, for the entire sequence of states leading to the tearing mode singularity in I. It may thus be concluded that secondary instabilities are not responsible for this singularity.

Insight into the nature of the singular tearing mechanism may be gained by comparing the rate of current diffusion, or the inverse island skin-time, to the reconnection rate. The former grows as the square of the island width, while the latter is simply proportional to this width. For narrow islands, the rate of current diffusion will thus be much larger than the rate of reconnection, allowing the current to remain in diffusive equilibrium during reconnection. In the strong tearing regime, by contrast, the relative rate of current diffusion slows until diffusive equilibrium can no longer be maintained. As the island continues to grow, its skin time eventually exceeds the reconnection time, causing the helicity to be progressively frozen into the plasma. In this respect, the singular tearing phenomenon is analogous to wavebreaking; It is caused by the dominance of the convective nonlinearity over the diffusive term as the island grows.

As a result of the restricted class of trial functions used in the neutral stability analysis, the possibility that a secondary instability may arise before the occurrence of the tearing singularity cannot be excluded. In order to account for the abrupt onset of kink-tearing growth, it is necessary for such a secondary instability to have a threshold at a finite island width. None of the currently known examples of secondary instabilities satisfies this requirement, as shown by Pritchett and Wu (1979) and Bondeson (1983) for the coalescence instability, and by Bussac et al. (1984) for the self-coalescence instability. 


\section{Appendix I: Asymptotic Expansions}

\section{A. Regularization of the $c_{l}^{\prime}$ Coefflcients}

The $c_{l}^{\prime}$ coefficients are defined by

$$
c_{l}^{\prime}(F)=\frac{d}{d F} \int_{-\pi}^{\pi} d \theta \frac{H(F-G(\theta))}{[2(F-G(\theta))]^{1 / 2}} \cos (l \theta),
$$

where $H(\cdot)$ is the Heaviside function. For $F>0$ this is simply

$$
c_{l}^{\prime}(F)=-\frac{1}{2} \int_{-\pi}^{\pi} d \theta \frac{\cos (l \theta)}{[2(F-G(\theta))]^{3 / 2}}
$$

For $F<0$, however, direct differentiation of the kernel leads to an ill-defined expression because of the turning-point singularity. This difficulty is resolved by integrating by parts:

$$
c_{l}^{\prime}(F)=2 \lim _{\iota \rightarrow 0}\left\{-\left[\frac{\cos (l \theta)}{d G / d \theta} \frac{H(F-G(\theta))}{[2(F-G(\theta))]^{1 / 2}}\right]_{0}^{\pi-\iota}+\int_{0}^{\pi-\iota} d \theta \frac{H(F-G(\theta))}{[2(F-G(\theta))]^{1 / 2}} \frac{d}{d \theta}\left(\frac{\cos (l \theta)}{d G / d \theta}\right)\right\} .
$$

It is convenient to rewrite the first term by using the identities

$$
\lim _{\ell \rightarrow 0}\left[\left(1-\frac{F-G(\theta)}{F-G(\pi)}\right) \frac{\cos (l \theta)}{d G / d \theta} \frac{H(F-G(\theta))}{[2(F-G(\theta))]^{1 / 2}}\right]_{0}^{\pi-\imath}=0
$$

and

$$
\begin{aligned}
& {\left[\frac{F-G(\theta)}{F-G(\pi)} \frac{\cos (l \theta)}{d G / d \theta} \frac{H(F-G(\theta))}{[2(F-G(\theta))]^{1 / 2}}\right]_{0}^{\pi-\ell} } \\
= & \int_{0}^{\pi-e} d \theta \frac{d}{d \theta}\left(\frac{[2(F-G(\theta))]^{1 / 2} \cos (l \theta)}{(F-G(\pi))(d G / d \theta)} H(F-G(\theta))\right) .
\end{aligned}
$$

After simplification, this leads to an integrable expression for $c_{1}^{\prime}$ :

$$
c_{l}^{\prime}(F)=2 \int_{0}^{\pi} d \theta \frac{H(F-G(\theta))}{[2(F-G(\theta))]^{1 / 2}}\left(\frac{\left(\sin (l \theta) \hat{G} G^{\prime}+\cos (l \theta)\left(\hat{G} G^{\prime \prime}-G^{2} / 2\right)\right.}{\left(F-G_{\min }\right) G^{\prime 2}}\right) .
$$

Where $G^{\prime}=d G / d \theta, \widehat{G}=G-G_{\min }$, and $G_{\min }=G(\pi)$. In the following sections this expression will be used to obtain expansions for the coefficients $c_{1}$ near the magnetic axis and separatrix. 


\section{B. Magnetic Axis}

The limiting value of $c_{l}$ at the magnetic axis is obtained by changing the integration variable to $\hat{G}=G-G_{\min }$ :

$$
c_{l}\left(G_{\min }+\epsilon\right)=2^{1 / 2} \int_{0}^{\epsilon} d \hat{G} \frac{d \theta}{d \hat{G}} \frac{\cos (l \theta(\hat{G}))}{(\epsilon-\hat{G})^{1 / 2}} .
$$

Expanding the argument in powers of $\hat{G}^{1 / 2}$ yields to lowest order $\theta(\hat{G})=\left(2 \hat{G} / G^{\prime \prime}(\pi)\right)^{1 / 2}$ and $\cos (1 \theta)=(-1)^{\prime}$. There follows

$$
c_{l}\left(G_{\min }+\epsilon\right)=\pi(-1)^{\prime}\left(G^{\prime \prime}(\pi)\right)^{-1 / 2}+O(\epsilon) .
$$

Similarly, changing variables and expanding the coefficient of the kernel in Eq. (A1) yields

$$
c_{l}^{\prime}\left(G_{\min }+\epsilon\right)=(-1)^{l+1}\left(G^{\prime \prime}(\pi)\right)^{-5 / 2}\left(4 l^{2} G^{\prime \prime}(\pi)+G^{\prime \prime \prime \prime}(\pi)\right) I / 2,
$$

where

$$
I=\int_{0}^{1} \frac{y^{2}}{\left(1-y^{2}\right)^{1 / 2}} d y=\frac{\pi}{4} .
$$

This procedure may be continued to show that $c_{l}$ has well-defined derivatives to arbitrary order in $l$.

\section{Separatrix}

The expansion procedure near the separatrix is similar to that employed near the magnetic axis, but since the integral now extends a finite distance away from the turning point it is preferable to avoid relying on a series expansion. The integral is separated instead into a singular part and a regularized remainder $\mathcal{R}$ :

$$
c_{l}(-\epsilon)=\left(-G^{\prime \prime}(0)\right)^{-1 / 2} \int_{G_{\min }}^{-\epsilon} d G \frac{1}{(-G)^{1 / 2}(-\epsilon-G)^{1 / 2}}+\mathcal{R},
$$

where

$$
\mathcal{R}=2^{1 / 2} \int_{G_{\min }}^{-\epsilon} d G \frac{1}{(-\epsilon-G)^{1 / 2}}\left(\frac{d \theta}{d G} \cos l \theta(G)-\frac{1}{\left(2 G^{\prime \prime}(0) G\right)^{1 / 2}}\right)
$$


For continuous, bounded $G(\theta)$ the coefficient in parentheses in $\mathcal{R}$ may be bounded uniformly by $A+B\left(G-G_{\min }\right)^{-1 / 2} ;$ there follows $|\mathcal{R}|<2 A\left(-2 G_{\min }\right)^{1 / 2}+2^{1 / 2} \pi B$. The integral of the singular part, by contrast, diverges logarithmically. Thus,

$$
c_{l}(-\epsilon) \sim-\left(-G^{\prime \prime}(0)\right)^{-1 / 2} \ln \epsilon+O(1)
$$

Similarly,

$$
c_{l}^{\prime}(-\epsilon) \sim-\left(-G^{\prime \prime}(0)\right)^{-1 / 2} \epsilon^{-1}-(1 / 2)\left(-G^{\prime \prime}(0)\right)^{-5 / 2}\left(l^{2} G^{\prime \prime}(0)+G^{\prime \prime \prime \prime}(0) / 3\right) \ln \epsilon+O(1) .
$$

These results may be substituted in the coefficients of the evolution equations. One finds

$$
\frac{c_{1}^{\prime}-c_{0}^{\prime}}{c_{0}^{\prime}} \sim\left(1 / 2 G^{\prime \prime}(0)\right) l^{2} \epsilon \ln \epsilon
$$

and

$$
\alpha_{k, l} \sim-(1 / 4)\left(-G^{\prime \prime}(0)\right)^{-5 / 2} k^{2} l^{2} \epsilon \ln ^{2} \epsilon
$$

The coefficients of the evolution equations are thus bounded and only mildly singular.

\section{Behavior Away from the Island}

The asymptotic behavior away from the island is easily obtained by expansion in inverse powers of $F$. The result is

$$
c_{l} \sim 2 \pi(2 F)^{-1 / 2}\left(\delta_{1,0}+G_{1} /(2 F)+O\left((G / F)^{2}\right)\right.
$$

where $\delta_{1,0}$ is the Kronecker symbol.

\section{E. Large Wavenumber Asymptotics}

For large wavenumber the trigonometric functions may no longer be expanded about the singular points. The integrals may nevertheless be evaluated by exploiting the property that the oscillations of the cosine caise the $c_{l}$ integrals to decay rapidly away from the singular points $F=G_{\min }$ and $F=0$. Near these points the integral is calculated as before by 
changing the integration variable to $\hat{G}$ and $G$ respectively, and expanding or regularizing the remaining functions. Near the magnetic axis one has

$$
c_{l}\left(G_{\min }+\epsilon\right) \sim(-1)^{\prime}\left(G^{\prime \prime}(\pi)\right)^{-1 / 2} 2^{1 / 2} \int_{0}^{\epsilon} d \hat{G} \frac{\cos \left[l\left(2 \hat{G} / G^{\prime \prime}(\pi)\right)^{1 / 2}\right]}{(\epsilon-\hat{G})^{1 / 2}}
$$

There follows

$$
c_{l}\left(G_{\min }+\epsilon\right) \sim \pi(-1)^{l}\left(G^{\prime \prime}(\pi)\right)^{-1 / 2} J_{0}\left(l\left(2 \epsilon / G^{\prime \prime}(\pi)\right)^{1 / 2}\right),
$$

where $J_{0}$ is the zeroth-order Bessel fuaction. The asymptotic expansion of $c_{l}$ near the separatrix may be obtained likewise. One finds

$$
c_{l}(-\epsilon) \sim \pi(-1)^{!}\left(G^{\prime \prime}(\pi)\right)^{-1 / 2} K_{0}\left(l\left(2 \epsilon / G^{\prime \prime}(\pi)\right)^{1 / 2}\right)
$$

where $K_{0}$ is the zeroth-order modified Bessel function. Eqs. (A6)-(A7) may be used to evaluate the matrix coefficients $a_{l, k}$ for large $l, k$ :

$$
a_{l, k}=\frac{4 l k}{\left(G^{\prime \prime}(\pi)\right)^{2}}\left(\frac{1}{c_{0}} \frac{\partial \psi}{\partial F}\right)_{F=G_{\min }} \int_{0}^{\infty} \frac{d y}{y} J_{1}(l y) J_{1}(k y),
$$

whence

$$
a_{l, k}=\frac{2(-1)^{l+k}}{\left(G^{\prime \prime}(\pi)\right)^{2}}\left(\frac{1}{c_{0}^{\prime}} \frac{\partial \psi}{\partial F}\right)_{F=G_{\min }} j_{<}^{2}
$$

where $j_{<}$is the smaller of the indices $l, k$. Note that the separatrix does not contribute to the integral at this order. This formula was used to check the convergence of the numerical integrations.

\section{Appendix II: Constant-Psi Limit}

Recall that the general thin island ordering is $w \sim \Delta^{\prime-1} \ll \lambda$. By inspection of Eqs.(5)(9), there follows $G \sim F \sim \psi \sim w^{2}, c_{l} \sim B_{* \theta} \sim w^{-1}$. The constant-psi approximation consists of the subsidiary ordering $w \Delta^{\prime} \ll 1$. As shown in Sec. III.D, this subsidiary ordering corresponds to islands with weak current perturbations, $I(\psi)=I_{0}+\tilde{I}$ where $\left.\tilde{I} / I_{0} \sim w\right\lrcorner^{\prime}$, 
and such that $G_{1} / I_{0}=\tilde{\psi}_{1}$. In this Appendix, the constant-psi subsidiary ordering is applied to the evolution equations.

The coefficients determining the reconnection rates are given to lowest order by

$$
a_{k, 1}^{(-1)}=I_{0}^{-1} \int_{G_{\min }}^{\infty} d F \alpha_{k, 1}
$$

with $\alpha_{k, 1}$ as before and

$$
b_{k}^{(0)}=-\eta \tilde{I}\left(1-(-1)^{k}\right) c_{0}\left(G_{\min }\right)+\int_{G_{\min }}^{\infty} d F \frac{c_{h}^{\prime}-c_{0}^{\prime}}{c_{0}} \frac{\partial}{\partial F}\left(\eta c_{0} \tilde{I}\right) .
$$

The magnitude of the reconnection rate is thus $d G_{1} / d t \sim \eta \tilde{I}$. Note that this is comparable to Rutherford's rate of reconnection. The evolution of the current-density, however, is generally more rapid. This is seen by expanding the flux distribution,

$$
\psi(F)=\psi\left(G_{\min }\right)+I_{0}^{-1}\left(F-G_{\min }\right)-I_{0}^{-2} \int_{G_{\min }}^{F} d F \tilde{I}(F)+O\left(G^{2}\right) .
$$

Substituting this expansion into Eq. (10) and taking the partial derivative with respect to $F$ yields

$$
\frac{\partial \tilde{I}}{\partial t}=I_{0}^{2} \frac{\partial}{\partial F}\left(\eta \tilde{I}\left(G_{\min }\right)-\frac{1}{I_{0}} \frac{d G_{\min }}{d t}+\frac{1}{I_{0}} \sum_{l=1}^{\infty} \frac{c_{l}^{\prime}-c_{0}^{\prime}}{c_{0}^{\prime}} \frac{d G_{l}}{d t}-\frac{1}{c_{0}^{\prime}} \frac{\partial}{\partial F}\left(\eta c_{0} \tilde{I}\right)\right) .
$$

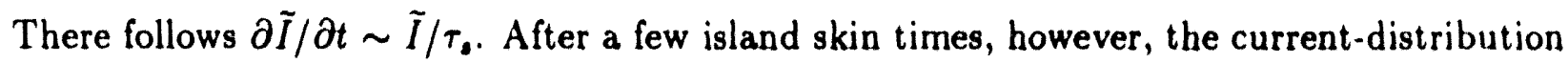
will relax into a diffusive quasi-equilibrium such that the right-hand side in the above expression vanishes. The relaxed current-density is then given by Eq. (12). It is not clear at first sight why this equation should be consistent with the evolution equation, Eq. (16). Recall, however, that Eq. (16) is obtained from Ohm's law and the equilibrium equation, and that the equilibrium equation is automatically satisfied to lowest order in the constant-psi regime. The compatibility of Eqs. (12) and (16) should thus be automatic to lowest order. It is nevertheless instructive to verify this explicitly. 
To verify the compatibility of Eqs. (12) and (16), the expression for the diffusively equilibrated current, Eq. (12), is substituted in Eq. (A9). There follows

$$
b_{k}^{(0)}=-\left(\dot{G}_{\min } / I_{0}\right)\left(1-(-1)^{k}\right) c_{0}\left(G_{\min }\right)+\frac{1}{I_{0}} \int_{G_{\min }}^{\infty} d F \frac{c_{k}^{\prime}-c_{0}^{\prime}}{c_{0}^{\prime}}\left(c_{l}^{\prime}-c_{0}^{\prime}\right) \dot{G}_{l} .
$$

By adding and subtracting the necessary terms this is

$$
b_{k}^{(0)}=\sum_{l=1}^{\infty} a_{k l} \dot{G}_{l}-\left(\dot{G}_{\min } / I_{0}\right)\left(1-(-1)^{k}\right) c_{0}\left(G_{\min }\right)+\frac{1}{I_{0}} \int_{G_{\min }}^{\infty} d F\left(c_{k \cdot l}^{\prime}-c_{k}^{\prime}-c_{l}^{\prime}+c_{0}^{\prime}\right) \dot{G}_{l} .
$$

Carrying out the integral, applying the boundary conditions (17)-(18), and using the identity $c_{k, l}^{\prime}\left(G_{\min }\right)=(-1)^{k+l} c_{0}^{\prime}\left(G_{\min }\right)$ yields the anticipated result,

$$
b_{k}^{(0)}=\sum_{l=1}^{\infty} a_{k l} \dot{G}_{l} .
$$

In order to obtain the constant-psi evolution equation, it is necessary to evalutate the next order terms in Eqs. (15)-(16). This requires first evaluating $\Omega^{(2)}(F)$ from Ohm's law. One finds

$$
\Omega^{(2)}(F)=-\frac{c_{l}^{\prime}-c_{0}^{\prime}}{c_{0}^{\prime}}\left(\frac{c_{l}}{c_{0}}\right) \frac{\dot{G}_{l} \dot{G}_{k}}{I_{0}^{2}}-\frac{\partial}{\partial t} \int_{G_{\min }}^{F} d \hat{F}\left(\frac{c_{l}}{c_{0}}\right) \frac{\dot{G}_{l}}{I_{0}^{2}}
$$

Substituting this result into Eq. (16), there follows after some algebra

$$
\frac{\pi}{2} \eta I_{0} \Delta_{l}^{\prime} \frac{d G_{l}}{d t}=\frac{d}{d t}\left(\int_{G_{\min }}^{\infty} d F \sum_{k=1}^{\infty} \frac{c_{k} c_{l}}{c_{0}} \frac{d G_{k}}{d t}\right)
$$

This can be recognized as the time derivative of Rutherford's evolution equation.

\section{Acknowledgments}

It is a pleasure to acknowledge helpful discussions with J. B. Taylor. This work was supported by a fellowship from the Center for Theoretical Physics at the University of Maryland, and by the United States Department of Energy. 


\section{References}

Aydemir, A. Y., Ross, D. W., and Wiley, J. C. 1989 Phys. Fluids B 1, 774.

Baty, H., Luciani, J. -F. and Bussac, M. -N. 1991 Nucl. Fusion 31, 2055.

Bernstein, I. B. Greene, J. M. and Kruskal, M. D. 1957 Phys. Rev. 108, 71.

Biskamp, D., 1991 Phys. Fluids B 3, 3353.

Bondeson, A. 1983 Phys. Fluids 26, 1275.

Bussac, M. N., Pellat, R., Soule, J. L., and Tagger, M. 1984 Phys. Lett. 105A, 51.

Bussac, M. N., Pellat, R. 1987 Phys. Rev. Lett. 59, 2650.

Coppi, B. and Friedlan I, A. B. 1971 Astrophys. J. 169, 379.

Coppi, B., Galvao, R., Rosenbluth, M. N., and Rutherford, P. H. 1976 Sov. J. Plasma Phys. 2, 1894.

Edery, D., Frey, M., Somon, J. P., Tagger, M., Soule, J. L., Pellat, R., and Bussac, M.-N. 1983 Phys. Fluids 26, 1165.

Edwards, A. W., Campbell, D. J., Engelhardt, W. W., Fahrbach, H.-U., Gill, R. D., Granetz, R. S. Tsuji, S. B., Tubbing, J. D., Weller, A., Wesson, J., and Zasche, D. 1986 Phys. Rev. Lett. 57, 210.

Finn, J. M., and Kaw, P. K. 1977 Phys. Fluids 20, 72.

Furth, H. P., Kileen, J., and Rosenbluth, M. N. 1963 Phys. Fluids 6, 459.

Furth, H. P., Rutherford, P. H., and Selberg, H. 1973 Phys. Fluids 16, 1054.

Grad, H. and Hogan, J. 1970 Phys. Rev. Lett. 24, 1337.

Irby, J. H., Drake, J. F., and Griem, H. R. 1979 Phys. Rev. Lett. 42, 228.

Jensen, T. H. and McClain, F. W. 1986 Phys. Fluids 29, 895.

Kadomtsev, B. B. 1975 Sov. J. Plasma Phys. 1, 389.

Kadomtsev, B. B. 1977 in Plasma Physics and Controlled Nuclear Fusion Research (IAEA, 
Vienna, 1977), Vol. I, p. 555.

Lee, J. K., Chu, M. S., Liu, C. S., and Drake, J. F. 1985 Phys. Fluids 28, 1585.

Lui, A. T. Y. 1987 in Magnetotail Physics, p. 101, Johns Hopkins Univ. Press, Baltimore.

Otani, N., and Strauss, H. R. 1988 Ap. J. 325, 468.

Park, W., Monticello, D. A., and White, R. B. 1984 Phys. Fluids 27, 137.

Pritchett, P. L., and Wu, C. C. 1979 Phys. Fluids 22, 2140.

Rutherford, P. H. 1973 Phys. Fluids 16, 1903.

Sato, T., Nakayama, Y., Hayashi, T., Watanabe, K. and Horiuchi, R. 1989 Phys. Rev. Lett.

63, 528 .

Steinolfson, R. S. and Van Hoven, G. 1983 Phys. Fluids 26, 117.

Steinolfson, R. S. and Van Hoven, G. 1984 Phys. Fluids 27, 1207.

Taylor, J. B. 1974 Phys. Rev. Lett. 35, 1139.

Taylor, J. B. 1975 Plasma Physics Note No. 10/75, Culham Laboratory.

Van Hoven, G. 1979 Astrophys. J. 232, 572.

Vasilyunas, V. M. 1975 Rev. Geophys. Space Phys. 13, 303.

Waelbroeck, F. L., 1989 Phys. Fluids B 1, 2372.

Waelbroeck, F. L. 1993 Phys. Rev. Lett. 70, 3259.

White, R. B. 1986 Rev. Mod. Phys. 58, 183. 


\section{Figure Captions}

1. Current-well $G$ and corresponding flux surfaces for the upper half of the island, showing the path of integration in the direction transverse to the island. 


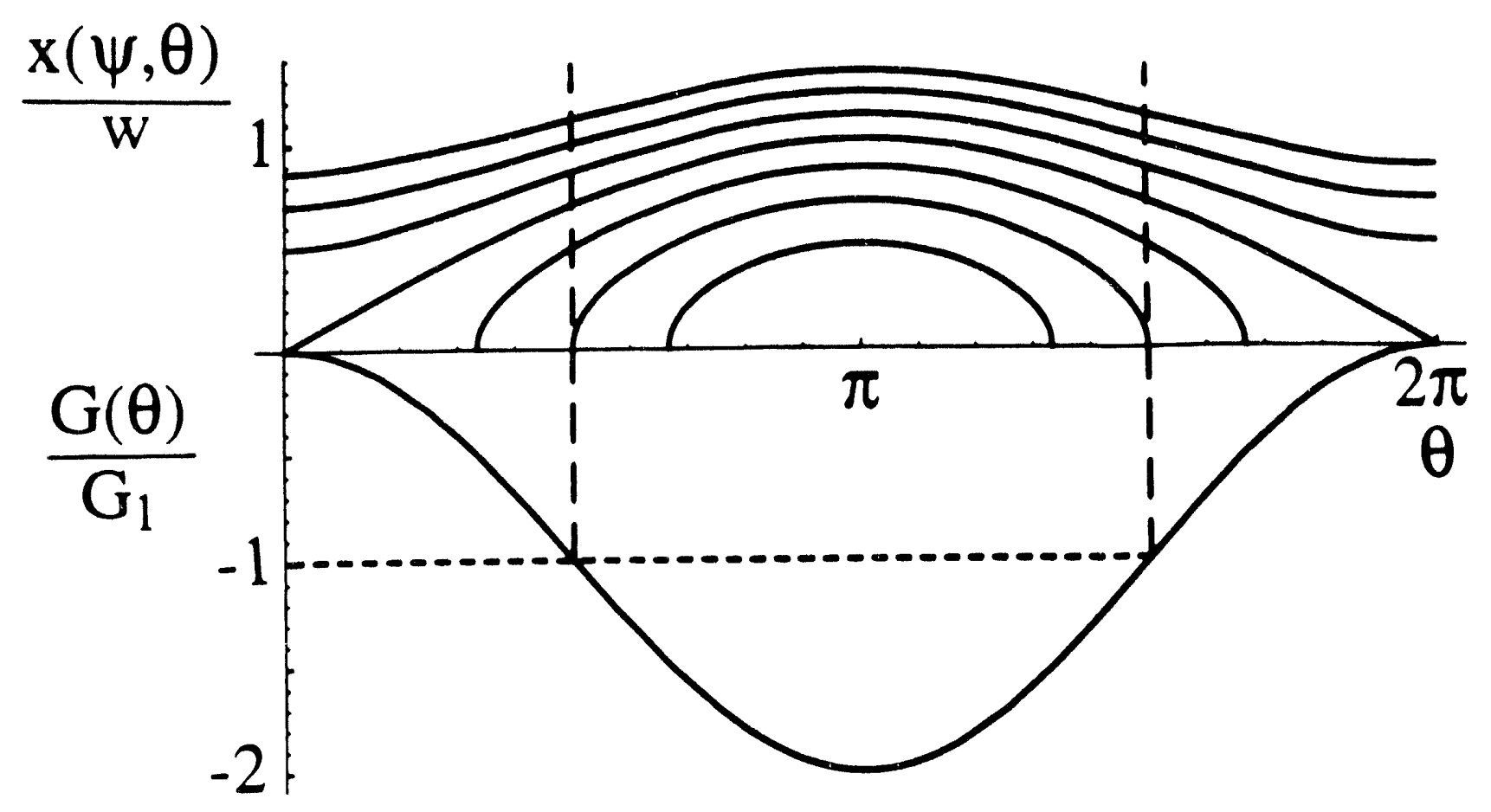

Fig. 1 

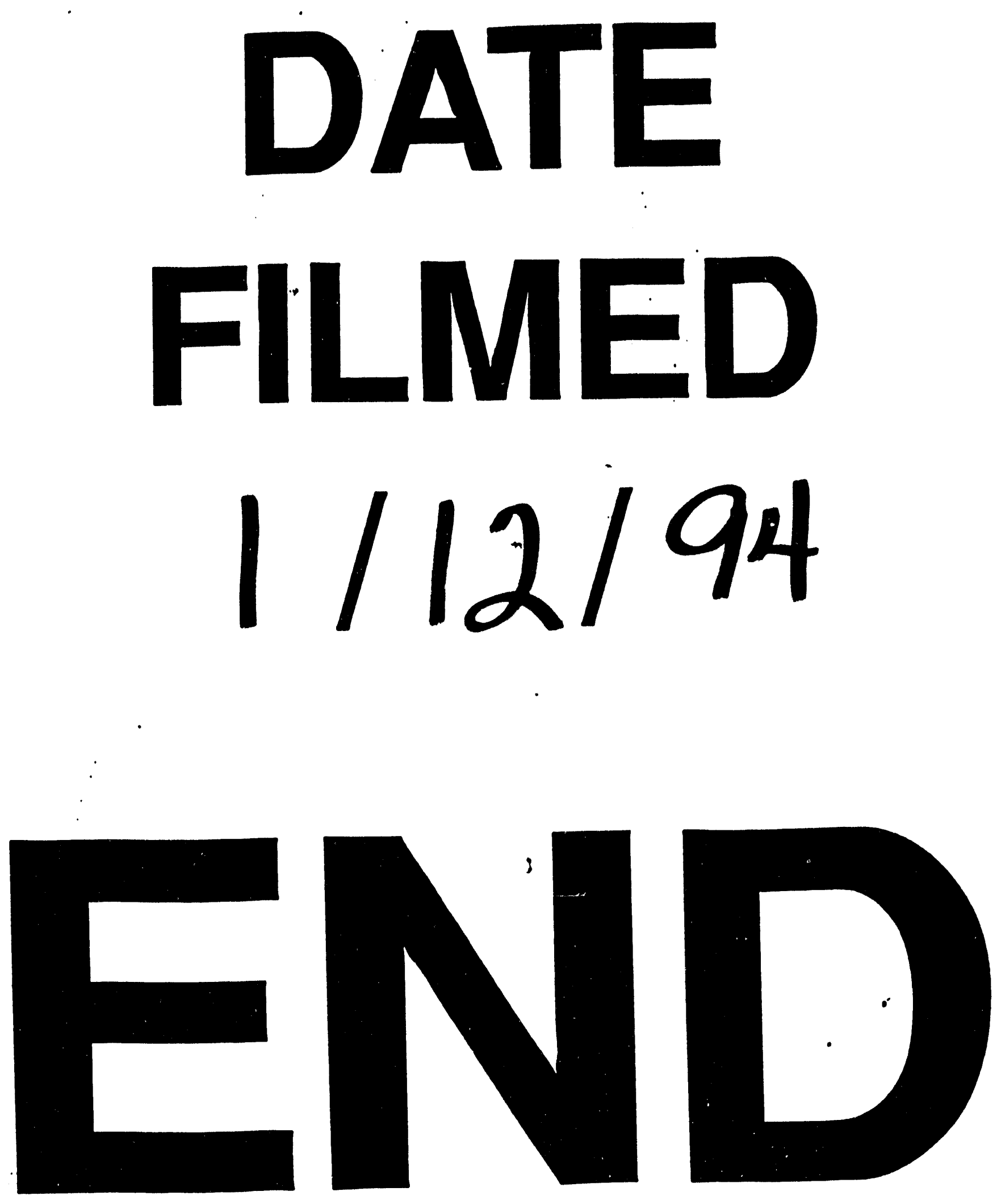


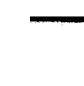

$$
\longrightarrow
$$

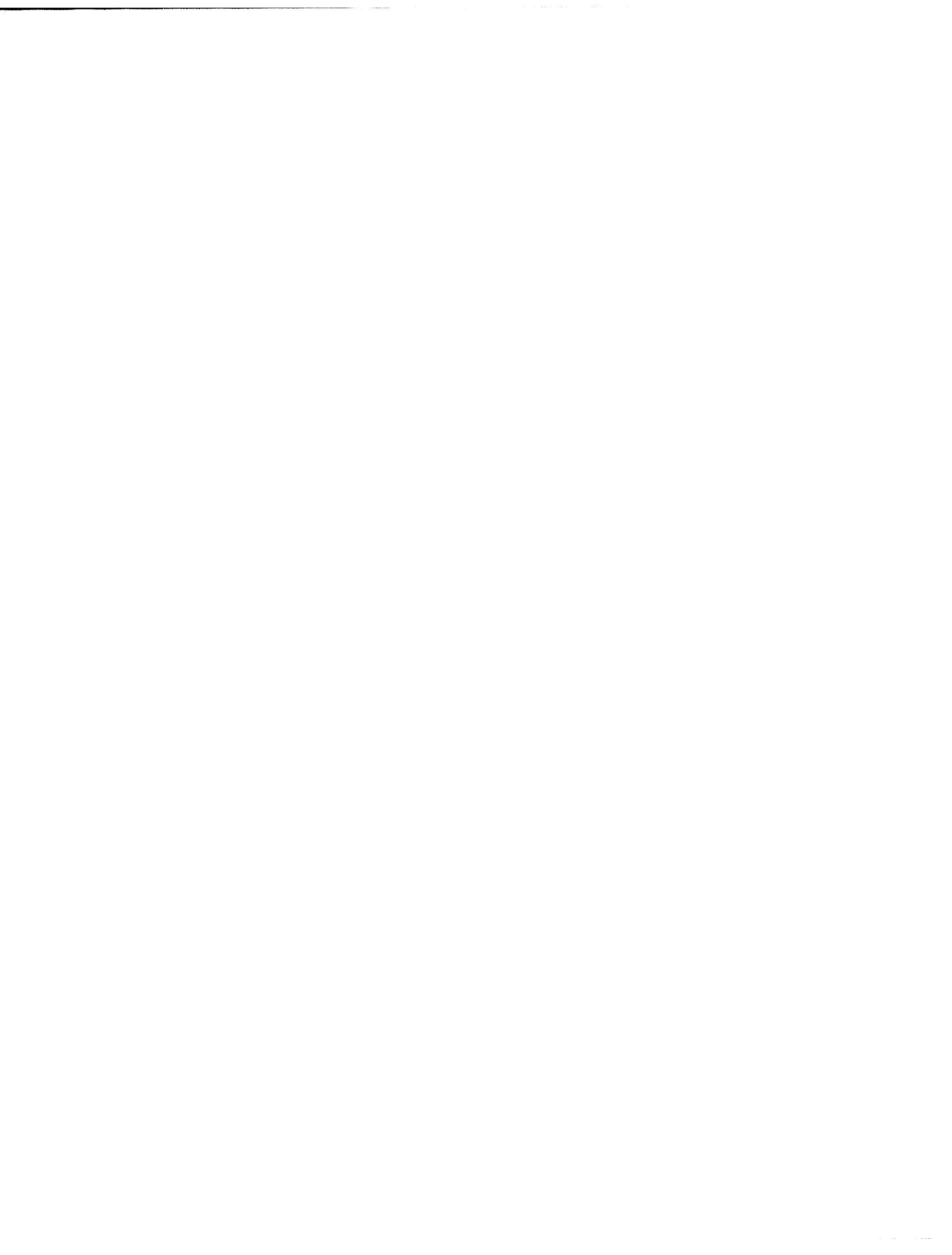

